\title{
Scaling of the dynamics of homogeneous states of one-dimensional long-range interacting systems
}

\author{
T. M. Rocha Filho, A. E. Santana, M. A. Amato, and A. Figueiredo \\ Instituto de Física and International Center for Condensed Matter Physics \\ Universidade de Brasília, CP: 04455, 70919-970 - Brasília, Brazil
}

\begin{abstract}
Quasi-Stationary States of long-range interacting systems have been studied at length over the last fifteen years. It is known that the collisional terms of the Balescu-Lenard and Landau equations vanish for one-dimensional systems in homogeneous states, thus requiring a new kinetic equation with a proper dependence on the number of particles. Here we show that the scalings discussed in the literature are mainly due either to small size effects or the use of unsuitable variables to describe the dynamics. The scaling obtained from both simulations and theoretical considerations is proportional to the square of the number of particles and a general form for the kinetic equation valid for the homogeneous regime is obtained. Numerical evidence is given for the Hamiltonian Mean Field and Ring models and a kinetic equation valid for the homogeneous state is obtained for the former system.
\end{abstract}

PACS numbers: 05.20.-y, 05.20.Dd, 05.10.Gg 
Classical systems with long range interaction can present unusual properties such as as non-ergodicity, anomalous diffusion, aging, non-Gaussian Quasi-Stationary States, negative microcanonical heat capacity and ensemble inequivalence, captured from the well known result of the positivity of the heat capacity in the canonical ensemble [1-10]. A pair interaction potential is said to be long ranged if it decays at long distances as $r^{-\alpha}$ with $\alpha \leq d$ where $d$ is the spatial dimension [5]. The dynamics of such systems has essentially three stages: (i) a violent relaxation [11, 12] even though a satisfactory theory is still lacking [9, 1315]), towards a Quasi Stationary State (QSS) in a short time roughly independent on the number of particles $N$; (ii) a QSS with a very long relaxation time to thermodynamic equilibrium that diverges with $N$, and finally, (iii) the system reaches the thermodynamic equilibrium. In the $N \rightarrow \infty$ limit the stage (iii) is never attained. After the first stage the system may also oscillate around a QSS with an amplitude decreasing with time due to a non-linear Landau damping [16]. The slowly varying state remains Vlasov stable and in some cases may loose its stability and rapidly evolve into another QSS, thereby resuming the slow evolution towards equilibrium [17]. This slow dynamics of the QSS have been extensively studied in the literature (see [1-4, 18, 25] and references therein) for different systems such as the Hamiltonian Mean Field (HMF) model [27 [32]:

$$
H=\sum_{i=1}^{N} \frac{p_{i}^{2}}{2}+\frac{1}{N} \sum_{i<j=1}^{N}\left[1-\cos \left(\theta_{i}-\theta_{j}\right)\right]
$$

with $\theta_{i}$ the position angle of particle $i$ on a circle and $p_{i}$ its conjugate momentum. Here we also investigate the dynamics of the homogeneous QSS for the Ring model with Hamiltonian [33]:

$$
H=\sum_{i=1}^{N} \frac{p_{i}^{2}}{2}+\frac{1}{N} \sum_{i<j=1}^{N} \frac{1}{\sqrt{2} \sqrt{1-\cos \left(\theta_{i}-\theta_{j}\right)+\epsilon}},
$$

where $\theta_{i}$ and $p_{i}$ have the same meanings as for the HMF model. Other systems with longrange interactions of interest, but not considered here, are one, two and three-dimensional self-gravitating systems discussed in [34 38], [39, 40] [41 44], respectively. The study of such systems along the same lines will be addressed in a future publication.

The first stage of the dynamics is described by the Vlasov equation (VE) which is satisfied by the one particle distribution function in the $N \rightarrow \infty$ limit [17, 45]. For finite $N$ this equation is valid only for short times encompassing the outburst of the violent relaxation. After this initial stage, collisional effects (graininess) accumulate and the VE must be corrected 
by considering higher order terms in an expansion in powers of $1 / N$ as it will be discussed below, leading to kinetic equations such as the Landau or Balescu-Lenard equations [46 48].

A sensible revision of the known kinetic equations for long-range interacting systems and their deductions, with all proper references, is presented by Chavanis in Refs. [49 52]. These equations usually can be obtained from the BBGKY hierarchy [48] by taking into account contributions from the two-body correlation functions, which are of order $1 / N$ [46, 49] that result in a time scale of collisional relaxation proportional to $N$ [49]. For three-dimensional gravity the dynamics scales as $N / \log N$, known as the Chandrasekhar scaling [53]. The Balescu-Lenard equation for a one-dimensional homogeneous system is written as [46]:

$$
\frac{\partial}{\partial t} f_{1}\left(p_{1} ; t\right)=2 \pi^{2} n \frac{\partial}{\partial p_{1}} \int \mathrm{d} p_{2} \int \mathrm{d} k \frac{k^{2} \tilde{V}(k)^{2}}{\left|\varepsilon\left(k, k p_{1}\right)\right|^{2}} \delta\left(k\left(p_{1}-p_{2}\right)\right)\left(\frac{\partial}{\partial p_{1}}-\frac{\partial}{\partial p_{2}}\right) f_{1}\left(p_{1} ; t\right) f_{1}\left(p_{2} ; t\right),
$$

where $f_{1}\left(p_{1} ; t\right)$ is the one-particle reduced distribution function, $n$ the particle density, $p_{i}$ the momentum of particle $i, \tilde{V}(k)$ the Fourier transform of the pair interaction potential and $\varepsilon\left(k, k p_{1}\right)$ is the dielectric function. Collective effects are ruled out if one takes $\varepsilon\left(k, k p_{1}\right)=1$ and that results in the Landau equation. The right-hand side of Eq. (3) vanishes identically due to the Dirac delta function [54, 55]. Therefore higher order terms must be kept when truncating the hierarchy, leading to a different scaling of the time evolution of a homogeneous state. More recently Sano proposed a derivation of a kinetic equation for one-dimensional homogeneous systems by summing contributions of all orders in the hierarchy [56]. Unfortunately his approach is limited to dilute gases and is not relevant for the problems addressed in this paper. It would be natural to expect that in the present case the predominant collisional corrections to the kinetic equation come from higher order terms proportional to $1 / N^{2}$. This implies a relaxation scaling proportional to $N^{2}$, as expected from a more straightforward application of kinetic theory such as that discussed by Chavanis in Refs. [49 52], even though he did not obtained a closed form kinetic equation at this order.

The $N^{2}$ scaling of the dynamics has previously been observed for one-dimensional neutral plasmas [58, 59]. However, different scalings proportional to $N^{1.7}$ and $\exp (N)$ were reported in Refs. [26], [27] and [28], respectively, for different types of initial conditions. The present authors obtained for the HMF model and different type of initial condition a scaling proportional to $N^{2}$ Ref. [17], which strongly suggests that the dominant contribution to the collisional term in the kinetic equation is given by the next term in the $1 / N$ expansion. A 
possible explanation for these discrepancies may originate from the fact that the number of particles in the simulations described in [17] are much greater than in [27] and [28], thereby a possible finite size effect should be carefully examined.

We argue that to properly probe how the dynamics depends on the number of particles a better choice of dynamical variables is to use higher moments of the velocity distribution, but keeping in mind that the second moment of $p$ is constant in the QSS due to energy conservation (homogeneity fixes the value of the magnetization and therefore of the potential energy up to small fluctuations). On the other hand, both this moment and the total magnetization can be used to determine the life-time of the homogeneous QSS given by the value of time at which their previously constant values start to change rapidly.

We stress the point that the magnetization is not a useful variable to follow the system dynamics (and therefore its scaling with $N$ ), as it depends only on the spatial distribution which is fixed in a homogeneous QSS.

In this paper one shows that the direct observation of the higher order moments of the velocity distribution leads to a different estimation of the scaling of the dynamics. We extend the calculations for the Ring model and show that the $N^{2}$ scaling is observed for homogeneous states of the systems described by this model. In this case, up to the authors knowledge, the scaling of the dynamics of the QSS for this model has not been studied due to the difficulty to pinpoint homogeneous QSS with a finite lifetime and the computation cost necessary for numerical simulations.

To corroborate our arguments (i) we have performed numerical simulations for the HMF model using the same type of initial conditions as considered in Refs. [27, 28] and varying the number of particles up to larger values than those encompassed in previous studies and, (ii) derive the kinetic equation to show that the relaxation scaling of the dynamics is proportional to $N^{2}$.

We note that the $\exp (N)$ scaling as claimed in Ref. [28] was obtained by conjecturing on the extrapolation of the dynamics of the phase of the magnetization vector, though it lacks more formal justification.

As explained above $M_{2}=\left\langle p^{2}\right\rangle$ is constant (up to fluctuations) in a homogeneous QSS, so we look at the fourth moment $M_{4}=\left\langle p^{4}\right\rangle$ of the momentum distribution starting from a homogeneous waterbag initial condition defined by $f(p ; 0)=1 / 2 p_{0}$ if $|p|<p_{0}$, and 0 otherwise $\left(p_{0}\right.$ constant). The moment $M_{4}$ varies slowly with time as a consequence of 
collisional corrections to the $\mathrm{VE}$, the dependence of the dynamics on $N$ being the inverse of that for the collisional term in the kinetic equation. All simulations were performed using a parallel implementation of a fourth-order symplectic integrator as described in Ref. [61].

In order to develop our approach, we show in Fig. 1 the numerical results for larger values of $N$, as the fluctuations turn out to be less important, and it can be seen that a $N^{2}$ scaling is yet more evident, while the $N^{1.7}$ scaling is clearly inappropriate. In Fig. 2 we show numerical results for the time evolution of $M_{4}$ for the same range of particle numbers $N$ as in Ref. [27] with two different time scalings: $N^{1.7}$ and $N^{2}$. Although the influence of fluctuations are important, a somewhat better data collapse is obtained for the $N^{2}$ scaling.

Figure 3 shows the observed lifetimes of the QSS for the initial conditions in Figs. 1 and 2 . The lifetimes are determined when the potential energy varies more than a given percentage (5\%) of its QSS value. In the left panel a scaling close to $N^{1.7}$ is obtained for $N<10000$ while the $N^{2}$ scaling is obtained for $N>10000$ in the right hand panel. Note also that the error bars for smaller $N$ are far from negligible such that a $N^{2}$ scaling is compatible with simulation data. This apparent discrepancy is explained by noting that the life-time of the homogeneous QSS is defined by the exact moment in time that the states looses its stability. Once the distribution function evolved closer to the stability threshold [17], fluctuations may trigger an instability and drive the system out of the QSS regime. Therefore fluctuations play an important role for small $N$ (they are of order $N^{-1 / 2}$ ), and thus generate a larger error bar for the life-times. In this case, the dynamics is governed by a kinetic equation with a $N^{2}$ scaling, while the life-time can in average deviate from this scaling as a consequence of larger fluctuations for small $N$.

To address the scaling $\exp (N)$ as presented in Ref. [28] for the HMF model we redo the same simulations under the same type of semi-elliptic initial conditions. Figure 4 shows the time evolution of $M_{4}$ for a time span greater than the lifetime of the QSS for $\epsilon=0.69$ and Fig. 5 shows results for $\epsilon=0.8$. The latter case corresponds to the same energy used in Ref. [28] and is such that the system remains always homogeneous up to the final thermodynamic equilibrium. Again a very good data collapse is obtained for the $N^{2}$ scaling for both energies.

We also consider here the scaling with time of the lifetime of homogeneous QSS for the Ring model. The time dependence of the kinetic energy for some values of $N$ and $\epsilon=0.1$ are shown in Fig. 6. We note that due to the QSS's being close to the stability threshold 

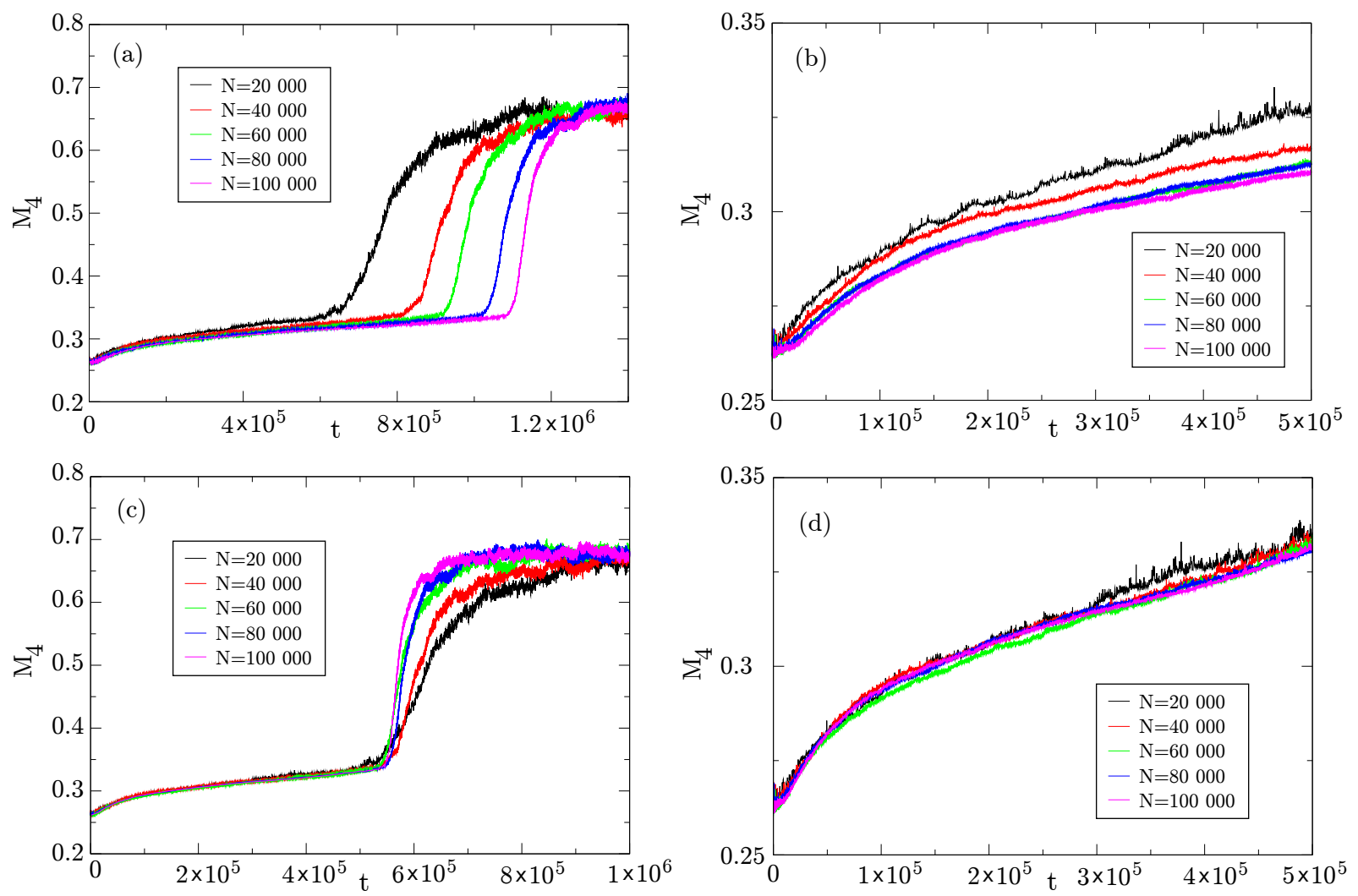

FIG. 1. (Color online) Moments $M_{4}$ as a function of time for the HMF model, with a homogeneous waterbag initial condition with energy per particle $e=0.69$ and $N=20000 ; 40000 ; 60000 ; 80000$; 100 000. a) Time is rescaled as $t \rightarrow t /\left(N \times 10^{-3}\right)^{1.7}$. b) Time window corresponding to the duration of the QSS with the same time scaling as (a). c) Time rescaled as $t \rightarrow t /\left(N \times 10^{-3}\right)^{2}$. d) Time window corresponding to the duration of the QSS with the same time scaling as (a). The graphics shows clearly that for larger number of particles the correct scaling is $N^{2}$.

fluctuations are more pronounced, but the $N^{2}$ scaling is nevertheless clearly observed.

All the numeric data presented here points out to a kinetic equation obtained from the next order correction in $N^{-1}$. To justify theoretically our findings we now briefly sketch how to obtain a kinetic equation for the simpler HMF system, leaving a more detailed discussion of a kinetic equation valid at the next order of approximation, either in a $1 / N$ expansion or in the weak coupling limit characterized by parameter $\alpha$ to a separate publication [57]. The 

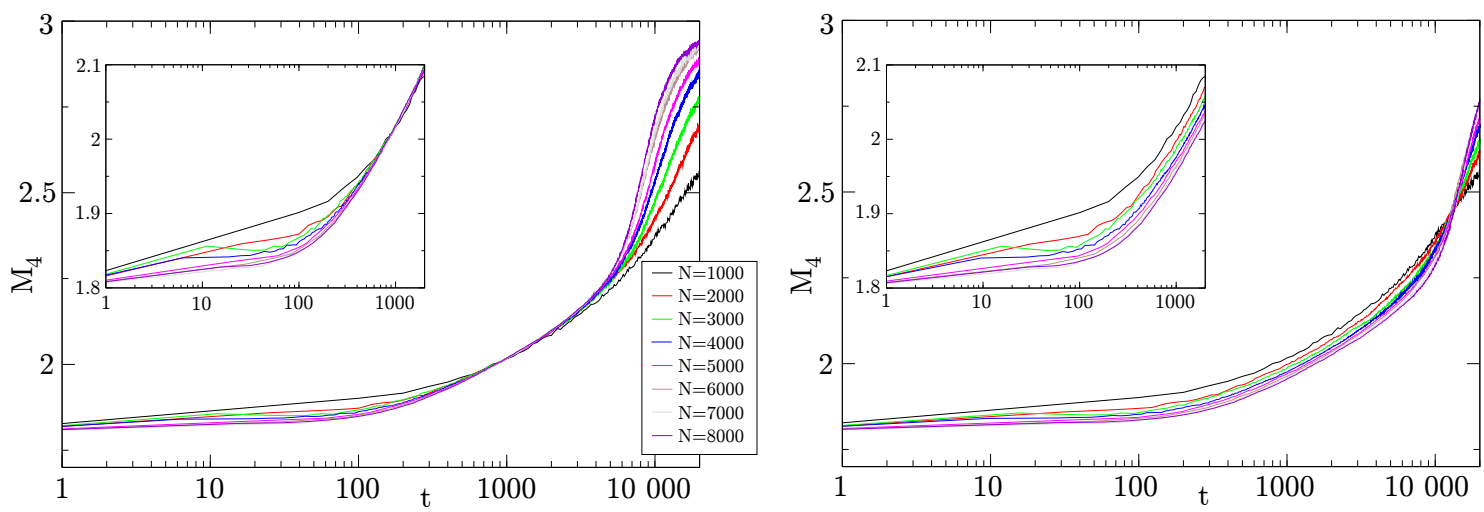

FIG. 2. (Color online) Moments $M_{4}$ as a function of time for the HMF model, with a homogeneous waterbag initial condition with energy per particle $e=0.69$ and small $N$ from 1000 up to 8000 , averaged over 100 realizations each. Left Panel: the time was rescaled as $t \rightarrow t /\left(N \times 10^{-3}\right)^{2}$. Right Panel: time rescaled as $t \rightarrow t /\left(N \times 10^{-3}\right)^{1.7}$. The insets are a zoom over the time interval where the system is in a homogeneous state.
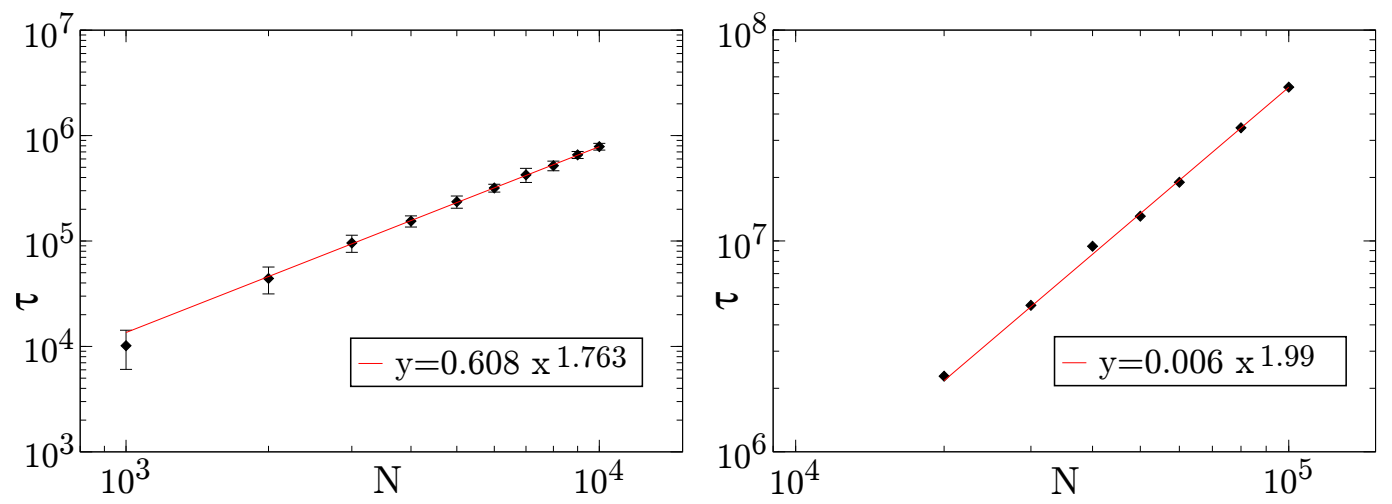

FIG. 3. (Color online) Left Panel: Life-times for the homogeneous waterbag state of the HMF model with energy per particle $e=0.69$ (solid circles) with respective error bars. The best fit yields a power law scaling $N^{1.76}$. Right Panel: same as the left panel but with greater number of particles and a best fit scaling of $N^{1.99}$. In this case the error bars are at most the size of the circles.

HMF system (as the ring model) belongs to a class of systems with generic Hamiltonian

$$
H(p, \theta)=\sum_{j=1}^{N} \frac{p_{j}^{2}}{2}+\frac{\alpha}{N} \sum_{j<k=1}^{N} V\left(\theta_{j}-\theta_{k}\right) .
$$



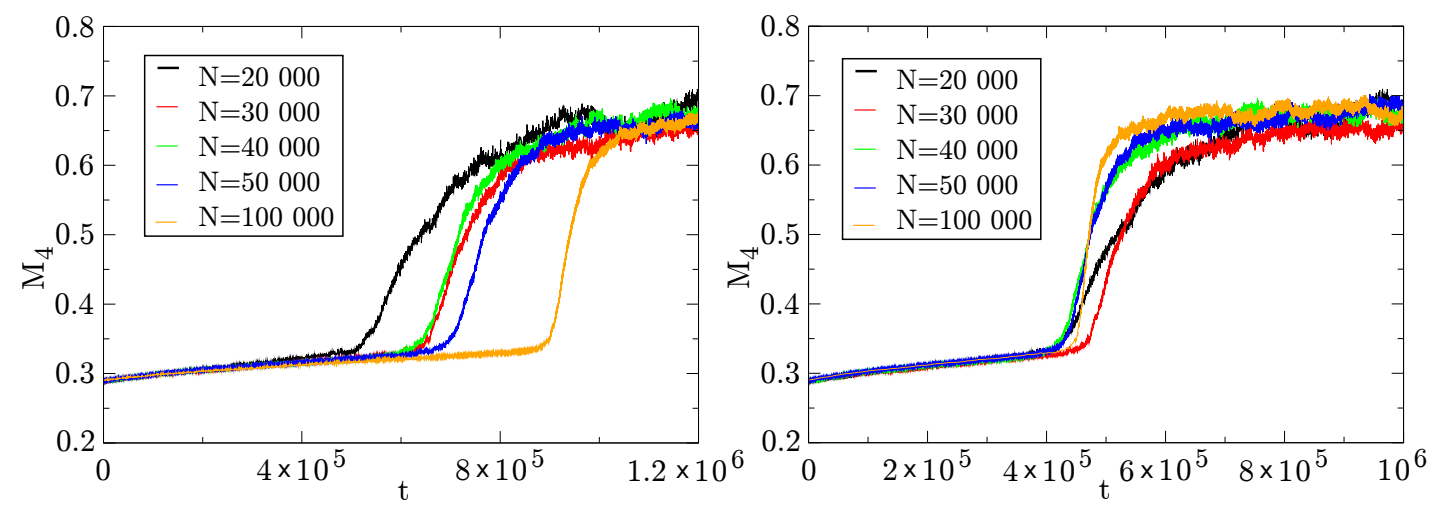

FIG. 4. (Color online) Moments $M_{4}$ as a function of time for the HMF model, with a homogeneous semi-elliptic initial condition with energy per particle $e=0.69$ and $N=20000 ; 30000 ; 40000$; 50 000; 100000 . Left Panel: the time was rescaled as $t \rightarrow t /\left(N \times 10^{-3}\right)^{1.7}$. Right Panel: time rescaled as $t \rightarrow t /\left(N \times 10^{-3}\right)^{2}$.

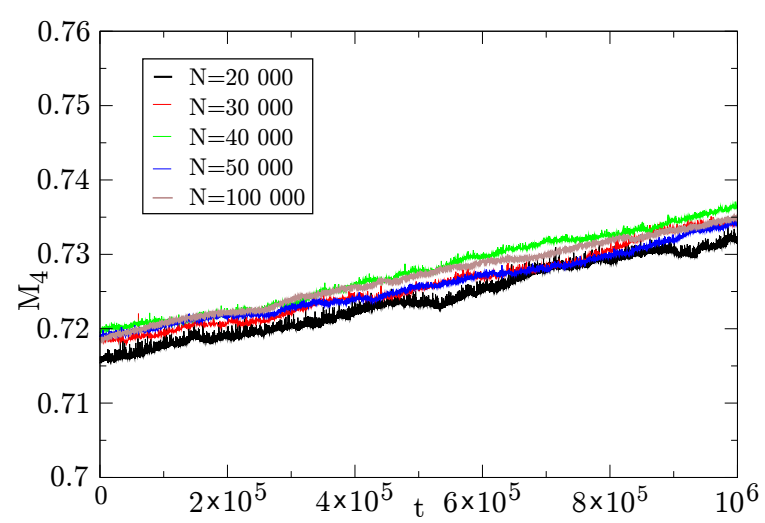

FIG. 5. (Color online) Fourth moment $M_{4}$ of the velocity distribution as a function of time for the HMF model, with a homogeneous semi-elliptic initial condition with energy per particle $e=0.8$ and $N=20000 ; 30000 ; 40000 ; 50000 ; 100000$. The time variable is rescaled as $t \rightarrow t /\left(N / 2 \times 10^{-4}\right)^{2}$ revealing the data collapse.

The pair potential interaction is rescaled by the Kac factor $1 / N$ in such a way that the energy is extensive (but non-additive) as for the Hamiltonians in Eqs. (1) and (2) 62]. The meaning of the dimensionless parameter $\alpha$ will be explained later in the paper. The kinetic equation for a homogeneous state can be obtained starting from the Liouville equation $\partial f_{N} / \partial t=$ $\left\{H, f_{N}\right\}$ for the $N$ particle distribution function in phase space $f_{N}\left(\theta_{1}, v_{1}, \ldots, \theta_{N}, v_{N} ; t\right)$ nor- 

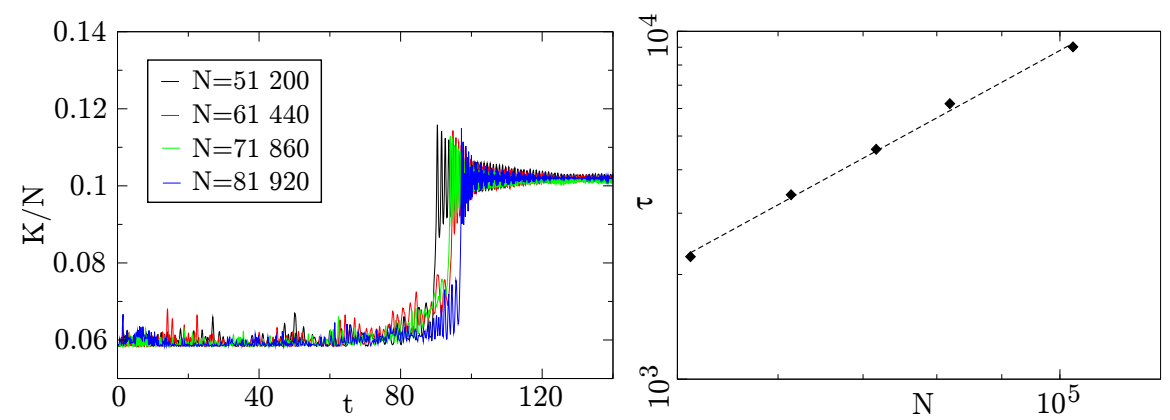

FIG. 6. Left panel: (Color online) Kinetic energy per particle for the ring model for a homogeneous waterbag initial condition with $p_{0}=0.59\left(f(p)=1 / 2 p_{0}\right.$ if $\left.-p_{0}<p<p_{0}\right)$ and $\epsilon=0.1$, for a few different numbers of particles. The time is rescaled as $t \rightarrow t /(N / 10240)^{2}$. Right panel: lifetimes for the QSS's in the left panel. The dashed line corresponds to the $N^{2}$ scaling.

malized to unity and $\left\{H, f_{N}\right\}$ is the Poisson bracket of $f_{N}$ with $H$. Defining the $s$-particle distribution function:

$$
f_{s} \equiv f_{s}\left(\theta_{1}, v_{1}, \ldots, \theta_{s}, v_{s} ; t\right)=\int \mathrm{d} \theta_{s+1} \mathrm{~d} v_{s+1} \cdots \mathrm{d} \theta_{N} \mathrm{~d} v_{N} f_{N}\left(\theta_{1}, v_{1}, \ldots, \theta_{N}, v_{N} ; t\right)
$$

the BBGKY hierarchy is obtained as [46, 48]:

$$
\frac{\partial}{\partial t} f_{s}=\sum_{j=1}^{s} \hat{K}_{j} f_{s}+\frac{1}{N} \sum_{j<k=1}^{s} \hat{\Theta}_{j k} f_{s}+\frac{N-s}{N} \sum_{j=1}^{s} \int \mathrm{d} \theta_{s+1} \mathrm{~d} v_{s+1} \hat{\Theta}_{j, s+1} f_{s+1}
$$

with

$$
\hat{K}_{j}=-v_{j} \frac{\partial}{\partial \theta_{j}}, \quad \hat{\Theta}_{j k}=-\frac{\partial}{\partial \theta_{j}} V\left(\theta_{j}-\theta_{k}\right) \partial_{j k}, \quad \partial_{j k} \equiv \frac{\partial}{\partial v_{j}}-\frac{\partial}{\partial v_{k}} .
$$

From this point onward we replace $p_{i}$ by $v_{i}$ (all particles have unit mass). In order to obtain a kinetic equation for the one-particle distribution function $f_{1}$ we must obtain $f_{2}$ as a functional of $f_{1}$. For that purpose we introduce the irreducible cluster representation (correlation expansion) for the reduced distribution functions $f_{s}$ up to $s=4$ as [46, 48]:

$$
\begin{aligned}
f_{2}(1,2)= & f_{1}(1) f_{1}(2)+C_{2}(1,2), \\
f_{3}(1,2,3)= & f_{1}(1) f_{1}(2) f_{1}(3)+\sum_{P(i, j, k)} f_{1}(i) C_{2}(j, k)+C_{3}(1,2,3), \\
f_{4}(1,2,3,4)= & f_{1}(1) f_{1}(2) f_{1}(3) f_{1}(4)+\sum_{P(i, j, k, l)}\left[f_{1}(i) C_{3}(j, k, l)+C_{2}(i, j) C_{2}(k, l)\right. \\
& \left.+f_{1}(i) f_{1}(j) C_{2}(k, l)\right]+C_{4}(1,2,3,4),
\end{aligned}
$$


where for simplicity the dependencies of each function on particle position and velocity is represented by the particle index, e. g. $C_{2}(1,2) \equiv C_{2}\left(\theta_{1}, \theta_{2}, v_{1}, v_{2}, t\right)$ and $\sum_{P(i, j, k)}$ stands for the sum over all different permutations between particles 1,2 and $3, f_{1}(1) \equiv f\left(v_{1}\right)$, $f_{2}(1,2) \equiv f_{2}\left(\theta_{1}-\theta_{2}, v_{1}, v_{2}\right), f_{3}(1,2,3) \equiv f_{2}\left(\theta_{1}-\theta_{2}, \theta_{2}-\theta_{3}, v_{1}, v_{2}, v_{2}\right)$ and similarly for the other terms. Since the reduced distributions are taken as fully symmetric by the permutation of any two particles, the same is valid for the pure correlation functions $C_{s}$.

Two-particle correlation requires the interaction of two particles, and therefore $C_{2}$ is of order $N^{-1}$. For three-particle correlations a two particle interaction between say particles 1 and 2 , and then between particles 2 and 3 are required, and $C_{3}$ is therefore of order $N^{-2}$, and so on. The Vlasov and Landau equations are obtained at order $N^{0}$ and $N^{-1}$, respectively. In order to explain our results and since the collision term vanishes at order $N^{-1}$, we must resort to the next order term proportional to $1 / N^{2}$. For a one-dimensional homogeneous system we obtain after replacing Eq. (8) into Eq. (6):

$$
\frac{\partial}{\partial t} f\left(v_{1}, t\right)=\int \mathrm{d} \theta_{2} \mathrm{~d} v_{2} \hat{\Theta}_{12} C_{2}\left(\theta_{1}, \theta_{2}, v_{1}, v_{2}, t\right) .
$$

In the equation above we dropped out the index in $f_{1}$, and in the subsequent equations. We now expand $C_{2}$ and $C_{3}$ in the form

$$
C_{2}=\frac{1}{N} C_{2}^{(1)}+\frac{1}{N^{2}} C_{2}^{(2)}+\mathcal{O}\left(\frac{1}{N^{3}}\right), \quad C_{3}=\frac{1}{N^{2}} C_{3}^{(2)}+\mathcal{O}\left(\frac{1}{N^{3}}\right) .
$$

Inserting the cluster expansion in Eqs. (8) and the expansions given at Eq. 12 into the hierarchy in Eq. (6) we obtain for $s=2$ at orders $1 / N$ and $1 / N^{2}$, respectively:

$$
\begin{gathered}
\left(\frac{\partial}{\partial t}+v_{1} \frac{\partial}{\partial \theta_{1}}+v_{2} \frac{\partial}{\partial \theta_{2}}\right) C_{2}^{(1)}\left(\theta_{12}, v_{1}, v_{2}, t\right)=V^{\prime}\left(\theta_{12}\right) \partial_{12} f\left(v_{1}, t\right) f\left(v_{2}, t\right) \\
+\frac{\partial}{\partial v_{1}} f\left(v_{1}, t\right) \int \mathrm{d} \theta_{3} \mathrm{~d} v_{3} V^{\prime}\left(\theta_{13}\right) C_{2}^{(1)}\left(\theta_{23}, v_{2}, v_{3}, t\right) \\
+\frac{\partial}{\partial v_{2}} f\left(v_{2}, t\right) \int \mathrm{d} \theta_{3} \mathrm{~d} v_{3} V^{\prime}\left(\theta_{23}\right) C_{2}^{(1)}\left(\theta_{13}, v_{1}, v_{3}, t\right) \\
\left(\frac{\partial}{\partial t}+v_{1} \frac{\partial}{\partial \theta_{1}}+v_{2} \frac{\partial}{\partial \theta_{2}}\right) C_{2}^{(2)}\left(\theta_{12}, v_{1}, v_{2}, t\right)= \\
-f\left(v_{1}, t\right) \int \mathrm{d} \theta_{3} \mathrm{~d} v_{3} V^{\prime}\left(\theta_{23}\right) \frac{\partial}{\partial v_{2}} C_{2}^{(1)}\left(\theta_{23}, v_{2}, v_{3}, t\right) \\
-2 \frac{\partial}{\partial v_{1}} f\left(v_{1}, t\right) \int \mathrm{d} \theta_{3} \mathrm{~d} v_{3} V^{\prime}\left(\theta_{13}\right) C_{2}^{(1)}\left(\theta_{23}, v_{2}, v_{3}, t\right) \\
+\frac{\partial}{\partial v_{1}} f\left(v_{1}, t\right) \int \mathrm{d} \theta_{3} \mathrm{~d} v_{3} V^{\prime}\left(\theta_{13}\right) C_{2}^{(2)}\left(\theta_{23}, v_{2}, v_{3}, t\right)
\end{gathered}
$$




$$
\begin{aligned}
& +\int \mathrm{d} \theta_{3} \mathrm{~d} v_{3} V^{\prime}\left(\theta_{23}\right) \frac{\partial}{\partial v_{2}} C_{3}^{(2)}\left(\theta_{12}, \theta_{23}, v_{1}, v_{2}, v_{3}, t\right)+1 \longleftrightarrow 2 \\
& +V^{\prime}\left(\theta_{12}\right) \partial_{12} C_{2}^{(1)}\left(\theta_{12}, v_{1}, v_{2}, t\right)
\end{aligned}
$$

with $1 \longleftrightarrow 2$ standing for permutation of particles 1 and 2 . For $s=3$ we obtain at the leading order:

$$
\begin{aligned}
& \left(\frac{\partial}{\partial t}+v_{1} \frac{\partial}{\partial \theta_{1}}+v_{2} \frac{\partial}{\partial \theta_{2}}+v_{3} \frac{\partial}{\partial \theta_{3}}\right) C_{3}^{(2)}\left(\theta_{12}, \theta_{23}, v_{1}, v_{2}, v_{3}, t\right)= \\
& \sum_{P(i, j, k)}\left[\partial_{i j} V^{\prime}\left(\theta_{i j}\right) C_{2}^{(1)}\left(\theta_{j k}, v_{j}, v_{k}, t\right)-f\left(v_{i}, t\right) \frac{\partial}{\partial v_{j}} f\left(v_{j}, t\right) \int \mathrm{d} \theta_{4} \mathrm{~d} v_{4} V^{\prime}\left(\theta_{j 4}\right) C_{2}^{(1)}\left(\theta_{k 4}, v_{k}, v_{4}, t\right)\right) \\
& +\frac{\partial}{\partial v_{i}} f\left(v_{i}, t\right) \int \mathrm{d} \theta_{4} \mathrm{~d} v_{4} V^{\prime}\left(\theta_{i 4}\right) C_{3}^{(2)}\left(\theta_{j k}, \theta_{k 4}, v_{j}, v_{k}, v_{4}\right) \\
& \left.+\frac{\partial}{\partial v_{i}} C_{2}^{(1)}\left(\theta_{i j}, v_{i}, v_{j}, t\right) \int \mathrm{d} \theta_{4} \mathrm{~d} v_{4} V^{\prime}\left(\theta_{j 4}\right) C_{2}^{(1)}\left(\theta_{j 4}, v_{j}, v_{4}, t\right)\right]
\end{aligned}
$$

In Eqs. 1315 we used the notation $\theta_{i j} \equiv \theta_{i}-\theta_{j}$ as for a homogeneous state $f_{s}$ and $C_{s}$ depend on position only through $\theta_{i j}$.

Equation 13 is and integro-differential equation for $C_{2}^{(1)}$. Noting that only the integral of $H_{2} \equiv \int \mathrm{d} v_{3} C_{2}^{(1)}$ is required in the kinetic integral, Lenard was able to solve the corresponding equation for $H_{2}$, but not for $C_{2}^{(1)}$ explicitly [47], which is required to solve the remaining equations (14) and (15). With some additional considerations on the strength of the interparticle force in a homogeneous state a closed form expression for the two and three-particle correlation functions can be obtained, as we proceed to show.

We observe that in a homogeneous state of the HMF model the force on a given particle is very small. Thus it is reasonable on a phenomenological ground to use some sort of weak coupling approximation to simplify the system of Eqs. 13 15). Indeed the mean-field force on each particle is given by $F=-V^{\prime} \propto(1 / N) \times N \times \alpha=\alpha$, i. e. the Kac factor times the particle number (from the sum over the remaining particles) with $\alpha$ characterizing the effective interaction strength in the homogeneous state which is proportional to the small magnitude of the fluctuations of the space distribution function around homogeneity. Such fluctuations, as illustrated in Fig. 7) diminishes with increasing $N$. In fact, we are considering that the approximation presented is a generalization of the Landau Equation for one-dimensional systems in a homogeneous state. In this way we have the following orders of magnitude: $C_{s} \propto \alpha^{s-1}$ and $V^{\prime} \propto \alpha$. In Eq. (13) the last two terms on the right-hand side can then be neglected, i. e. we keep terms up to order $\alpha$ and neglect terms of order $\alpha^{2}$. The 

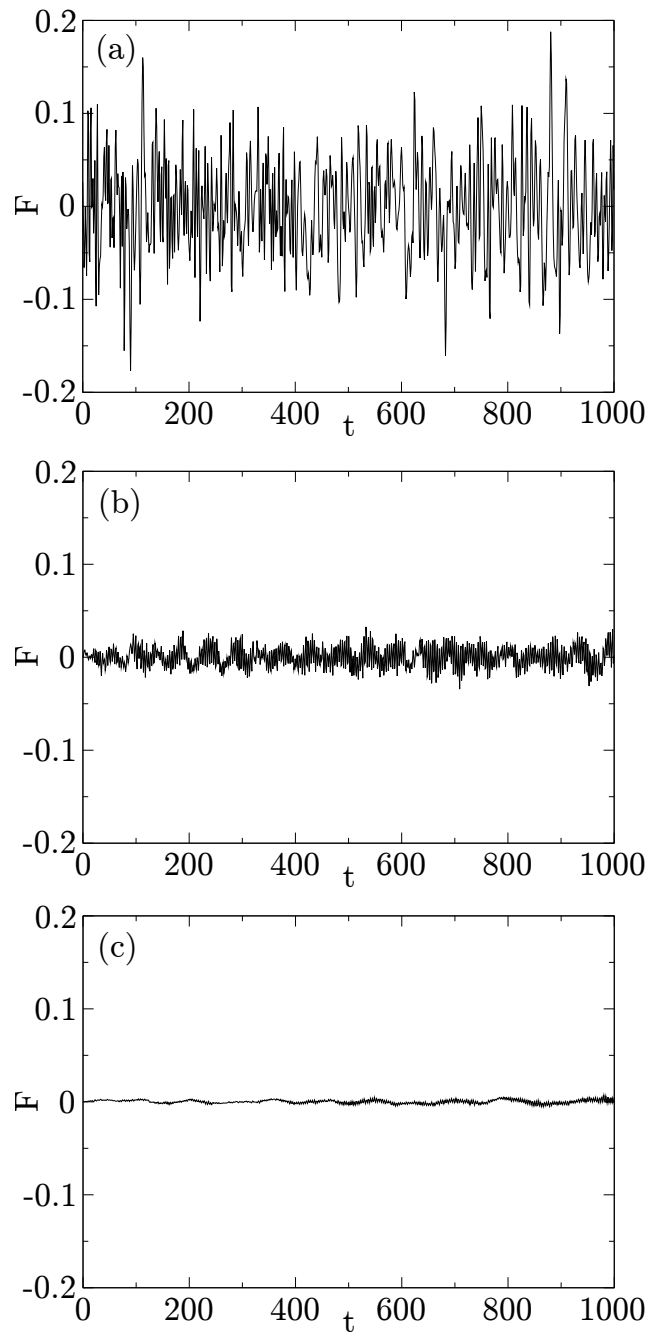

FIG. 7. Force on a single particle for a) $N=1000$, b) $N=10000$ and c) $N=10000000$ with energy per particle $e=0.69$.

third term in the summation in the right-hand side of Eq. 15 is of order $\alpha^{3}$ and is also negligible.

Since the physical space of the HMF model is periodic, it is convenient to introduce the Fourier series (discarding for convenience the constant term in $V$ ):

$$
\begin{aligned}
& V\left(\theta_{12}\right)=\sum_{n} \tilde{V}(n) \mathrm{e}^{i n \theta_{12}}, \quad C_{2}\left(\theta_{12}, v_{1}, v_{2} ; t\right)=\sum_{n} \tilde{C}_{2}\left(k, v_{1}, v_{2} ; t\right) \mathrm{e}^{i n \theta_{12}} \\
& C_{3}\left(\theta_{12}, \theta_{23}, v_{1}, v_{2} ; t\right)=\sum_{n, m} \tilde{C}_{3}\left(n, m, v_{1}, v_{2} ; t\right) \mathrm{e}^{i n \theta_{12}} \mathrm{e}^{i m \theta_{23}}
\end{aligned}
$$


with $n, m$ integers ranging from $-\infty$ to $\infty$ and

$$
\begin{aligned}
& \tilde{V}(n)=\frac{1}{2 \pi} \int \mathrm{d} \theta_{12} V\left(\theta_{12}\right) \mathrm{e}^{-i n \theta_{12}}=-\frac{1}{2}\left[\delta_{n, 1}+\delta_{n,-1}\right], \\
& \tilde{C}_{2}\left(n, v_{1}, v_{2} ; t\right)=\frac{1}{2 \pi} \int \mathrm{d} \theta_{12} C_{2}\left(\theta_{12}, v_{1}, v_{2} ; t\right) \mathrm{e}^{-i n \theta_{12}}, \\
& \tilde{C}_{3}\left(n, m, v_{1}, v_{2}, v_{3} ; t\right)=\frac{1}{(2 \pi)^{2}} \int \mathrm{d} \theta_{12} \mathrm{~d} \theta_{23} C_{2}\left(\theta_{12}, \theta_{23}, v_{1}, v_{2}, v_{3} ; t\right) \mathrm{e}^{-i n \theta_{12}} \mathrm{e}^{-i n \theta_{23}},
\end{aligned}
$$

where $\delta_{n, m}$ is the Kronecker delta. Note that $C_{4}$ is neglected at this approximation as it is of order $1 / N^{3}$. Equation 11 is rewritten as

$$
\begin{aligned}
\frac{\partial}{\partial t} f\left(v_{1} ; t\right) & =i \int \mathrm{d} v_{2} \sum_{n} n \tilde{V}(n) \partial_{12} \tilde{C}_{2}\left(n, v_{1}, v_{2} ; t\right) \\
& =\frac{i}{2} \frac{\partial}{\partial v_{1}} \int \mathrm{d} v_{2}\left[\tilde{C}_{2}\left(-1, v_{1}, v_{2}, t\right)-C_{2}\left(1, v_{1}, v_{2}, t\right)\right] \\
& =\frac{\partial}{\partial v_{1}} \int \mathrm{d} v_{2} \operatorname{Im}\left[C_{2}\left(1, v_{1}, v_{2}, t\right)\right]
\end{aligned}
$$

where we used the property $C_{2}\left(-1, v_{1}, v_{2}, t\right)=C_{2}\left(1, v_{1}, v_{2}, t\right)^{*}$ as it is the Fourier coefficient of a real function. Thus only the imaginary part of the Fourier transform $\tilde{C}_{2}$ contributes to the kinetic equation.

Using the Fourier series in Eq. (16) and (17) as well as the Fourier coefficients for the HMF potential yields:

$$
\left(\frac{\partial}{\partial t}+i v_{12} m\right) \tilde{C}_{2}^{(1)}\left(m, v_{1}, v_{2}, t\right)=-\frac{i}{2}\left(\delta_{m, 1}-\delta_{m,-1}\right) \partial_{12} f\left(v_{1}, t\right) f\left(v_{2}, t\right),
$$

with $v_{j k} \equiv v_{j}-v_{k}$ and $\tilde{C}_{n}^{(s)}$ the Fourier coefficient of $C_{n}^{(s)}$. Before solving Eq. 19 we must determine the time evolution of the one-particle distribution function, which is given by the ballistic approximation (free motion with constant velocity), valid up to order $1 / N^{2}$ in the present case $f(v, t)=f(v)+\mathcal{O}\left(N^{-2}\right)$. The solution of Eq. 19 is now easily obtained as:

$$
\begin{aligned}
\tilde{C}_{2}^{(1)}\left(m, v_{1}, v_{2}, t\right)= & \tilde{C}_{2}^{(1)}\left(m, v_{1}, v_{2}, 0\right) \mathrm{e}^{-i v_{12} m t} \\
& -\frac{i}{2}\left(\delta_{m, 1}-\delta_{m,-1}\right) \int_{0}^{t} \mathrm{~d} \tau \mathrm{e}^{i v_{12} m \tau} \partial_{12} f\left(v_{1}, \tau\right) f\left(v_{2}, \tau\right) .
\end{aligned}
$$

The first term on the right-hand side is a transient that describes the memory of the initial correlation and rapidly becomes negligible (see [46] for a detailed discussion of this point). On the other hand, the integral in $\tau$ tends to the Cauchy integral for large $t$ [46]:

$$
\int_{0}^{\infty} \mathrm{d} t \mathrm{e}^{i v_{12} m t}=\pi \delta\left(v_{12} m\right)+i \mathcal{P}\left(\frac{1}{v_{12} m}\right),
$$


with $\mathcal{P}(1 / a)$ the principal part of $1 / a$ and $\delta(a)$ the Dirac delta function. This last step is a Markovianization procedure of the solution for the correlation function. Therefore, the solution of Eq. (13) takes the form:

$$
\tilde{C}_{2}^{(1)}\left(m, v_{1}, v_{2}, t\right)=\frac{1}{2} \mathcal{P}\left(\frac{1}{v_{12} m}\right)\left(\delta_{m, 1}-\delta_{m,-1}\right) \partial_{12} f\left(v_{1}, t\right) f\left(v_{2}, t\right) .
$$

In Eq. (14) all terms must be kept. Indeed if we keep only terms of order $\alpha^{2}$ this would imply discarding all terms containing $\tilde{C}_{3}^{(2)}$. It can be shown that the next order correction $\tilde{C}_{2}^{(2)}$ would be real (see for instance the appendix in Ref. [47]), not contributing to the kinetic equation. Consequently the next order non-vanishing correction to the kinetic equation comes from the contribution of three-particle correlations. The later can be determined by solving Eq. 15 neglecting terms of order $\alpha^{3}$, i. e. the last two terms between brackets on the right-hand side. Both remaining equations Eqs. (14) and (15) are then written in Fourier space, and the latter solved for $\tilde{C}_{3}^{(2)}$. This solution is then replaced in Eq. 14 and that is solved for $\tilde{C}_{2}^{(2)}$, which together with Eq. 18) yield the desired kinetic equation. All these calculations are straightforward but quite long and tedious, but easily handled using a computer algebra system [63] with specific routines developed by the authors for this purpose and is given in Supplemental Material at [URL to be inserted by publisher]. Here we only show the final result for $\operatorname{Im} \tilde{C}_{2}^{(2)}$ obtained as delineated above:

$$
\begin{aligned}
& \operatorname{Im} \tilde{C}_{2}(1, v 1, v 2, t)=\frac{1}{N^{2}} \operatorname{Im} \tilde{C}_{2}^{(2)}(1, v 1, v 2, t)=\frac{\pi^{2}}{4} \mathcal{P}\left(\frac{1}{v_{1}-v_{2}}\right) \\
\times & \left.\left\{\mathcal{P}^{\prime}\left(\frac{1}{v_{1}-v_{2}}\right) \hat{\mathcal{K}}_{I}+\mathcal{P}^{\prime \prime}\left(\frac{1}{v_{1}-v_{2}}\right) \hat{\mathcal{K}}_{I I}\right\} f\left(v_{1}\right) f\left(v_{2}\right) f\left(v_{3}\right)\right|_{v_{3}=2 v_{2}-v_{1}}+1 \longleftrightarrow 2,
\end{aligned}
$$

where $1 \longleftrightarrow 2$ stands for terms obtained from a permutation of particles 1 and 2 , and

$$
\begin{aligned}
& \hat{\mathcal{K}}_{I} \equiv 3 \frac{\partial}{\partial v_{2}} \frac{\partial}{\partial v_{3}}-2 \frac{\partial^{2}}{\partial v_{3}^{2}}-2 \frac{\partial}{\partial v_{1}} \frac{\partial}{\partial v_{3}}-\frac{\partial}{\partial v_{1}} \frac{\partial}{\partial v_{2}}+2 \frac{\partial^{2}}{\partial v_{2}^{2}}, \\
& \hat{\mathcal{K}}_{I I} \equiv-2 \frac{\partial}{\partial v_{2}}+\frac{\partial}{\partial v_{1}}+\frac{\partial}{\partial v_{3}} .
\end{aligned}
$$

Equations (18) and (23) give the final form of the kinetic equation for the HMF model in a homogeneous state. The collisional integral is non-vanishing and proportional to $1 / N^{2}$ explaining, from a theoretical viewpoint, the scale $N^{2}$ for the dynamics of one-dimensional homogeneous systems, as shown in all the simulations reported in this paper. Our results are at variance with previous results reported in the literature, and its origin may be understood as an effect of the size of $N$ on those calculations. In Ref. 60] Ettoumi and Firpo developed a 
stochastic theory based on the first passage time approach and taking into account the role of fluctuations. Their approach nevertheless requires the numeric determination of a diffusion coefficient and is therefore limited to the number of particles considered (up to $N=20000$ ). These authors were able to obtain the exponent 1.7, and it would be interesting to extend their work to larger number of particles as they have been considered here, where a $N^{2}$ scaling is expected. Our results strongly suggest that although the dynamics, as represented here by the time evolution of the velocity moments, scales as $N^{2}$, even for small $N$, but when the effect of fluctuations is taken into account in the destabilization of the QSS, a different exponent can be obtained. Further research in course should clarify these points. As a concluding remark, in the present paper we show that the use of the magnetization as relevant dynamical variable only depends on the space fluctuations around homogeneity (the magnetization is always zero up to fluctuations). On the other hand, the dynamics in the velocity distribution, which changes over time according to the kinetic equation, is not probed. The statistical moments of the velocity distribution are therefore more suitable variables to describe the dependence of the dynamics on the particle number $N$.

The authors acknowledge partial financial support by CAPES and CNPq (Brazilian agencies). TMRF would like to thank Bruno Marcos for fruitful discussions.

[1] A. Campa, T. Dauxois and S. Ruffo, Phys. Rep. 480, 57 (2009).

[2] Dynamics and Thermodynamics of Systems with Long-Range Interactions, T. Dauxois, S. Ruffo, E. Arimondo and M. Wilkens Eds. (Springer, Berlin, 2002).

[3] Dynamics and Thermodynamics of Systems with Long-Range Interactions: Theory and Experiments, A. Campa, A. Giansanti, G. Morigi and F. S. Labini (Eds.), AIP Conf. Proceedings Vol. 970 (2008).

[4] Long-Range Interacting Systems, Les Houches 2008, Session XC, T. Dauxois, S. Ruffo and L. F. Cugliandolo Eds. (Oxford Univ. Press, Oxford, 2010).

[5] T. Dauxois, S. Ruffo, E. Arimodo and M. Wilkens, Dynamics and Thermodynamics of Systems with Long-Range Interactions: An Introduction, in [2].

[6] T. M. Rocha Filho, M. A. Amato and A. Figueiredo, J. Phys. A 42, 165001 (2009).

[7] T. M. Rocha Filho, M. A. Amato, and A. Figueiredo, Phys. Rev. E 85, 062103 (2012). 
[8] T. M. Rocha Filho, A. Figueiredo and M. A. Amato, Phys. Rev. Lett. 95, 190601 (2005).

[9] A. Figueiredo, T. M. Rocha Filho and M. A. Amato, Europhys. Lett. 83, 30011 (2008).

[10] A. Figueiredo, Z. T. Oliveira Jr, T. M. Rocha Filho, R. Matsushita and M. A. Amato, condmat.stat-mech:1208.4878.

[11] D. Lynden-Bell, Mon. Not. R. Astr. Soc. 136, 101 (1967).

[12] This term was originally coined by LyndenBell who first developed a statistical theory for predicting its outcome.

[13] I. Arad and D. Lynden-Bell, Mon. Not. R. Astr. Soc. 361, 385 (2005).

[14] I. Arad and P. Johansson, Mon. Not. R. Astr. Soc. 362, 252 (2005).

[15] P. de Buyl and P. Gaspard, Phys. Rev. E 84, 061139, (2011).

[16] C. Mouhot and C. Villani, Acta Math. 207, 29 (2011).

[17] T. M. Rocha Filho, A. E. Santana, J. R. Steiner, M. A. Amato and A. Figueiredo, condmat.stat-mech:1305.2903.

[18] F. Baldovin and E. Orlandini, Phys. Rev. Lett. 97, 100601 (2006).

[19] P.-H. Chavanis, Physica A 365, 102 (2006).

[20] P. de Buyl, D. Fanelli, R. Bachelard and G. De Ninno, Phys. Rev. Sep. Top. Acc. Beams 12, 060704 (2009).

[21] A. Gabrielli, M. Joyce and B. Marcos, Phys. Rev. Lett. 105, 210602 (2010).

[22] S. Gupta and D. Mukamel, J. Stat. Mech P03015 (2011).

[23] M. Assllani, D. Fanelli, A. Turchi, T. Carletti and X. Leoncini, Phys. Rev. E 85, 021148 (2012).

[24] R. Pakter and Y. Levin, Phys. Rev. Lett. 110, 140601 (2013).

[25] R. Bachelard, C. Chandre, D. Fanelli, X. Leoncini and S. Ruffo, Phys. Rev. Lett. 101, 260603 (2008).

[26] D. H. Zanette and M. A. Montemurro, Phys. Rev. E 67, 031105 (2003).

[27] Y. Y. Yamaguchi, J. Barré, F. Bouchet, T. Dauxois and S. Ruffo, Physica A 337, 36 (2004).

[28] A. Campa, A. Giansanti and G. Morelli, Phys. Rev. E 76, 041117 (2007).

[29] A. Campa, P. H. Chavanis, A. Giansanti and G. Morelli, Phys. Rev. E 78, 040102(R) (2008).

[30] K. Jain, F. Bouchet and D. Mukamel, J. Stat. Mech. P11008 (2007).

[31] P. de Buyl, cond-mat.stat-mech:1210.6316.

[32] M. Antoni and S. Ruffo, Phys. Rev. E 52, 2361 (1995). 
[33] Y. Sota, O. Iguchi, M. Morikawa, T. Tatekawa and K. I. Maeda, Phys. Rev. E 64, 056133 (2001).

[34] P. Valageas, Phys. Rev. E 74,016606 (2006).

[35] M. Joyce and T. Worrakitpoonpon, J. Stat. Mech. P10012 (2010).

[36] S. Gupta and D. Mukamel, J. Stat. Mech. P08026 (2010).

[37] S. Gupta and D. Mukamel, J. Stat. Mech. P03015 (2011).

[38] S. Gupta and D. Mukamel, Phys. Rev. Lett. 105, 040602 (2010).

[39] B. Marcos, cond-mat.stat-mech:1212.0959.

[40] T. N. Teles, Y. Levin, R. Pakter, and F. B. Rizzato, J. Stat. Mech. P05007 (2010).

[41] J. Diemand, B. Moore, J. Stadel and S. Kazantzidis, Mon. Not. Roy. Astron. Soc. 348, 977 (2004).

[42] A. Gabrielli, M. Joyce, and B. Marcos, Phys. Rev. Lett. 105, 210602 (2010).

[43] S. Chandrasekhar, Principles of stellar dynamics (University of Chicago Press, 1942).

[44] J. Heyvaerts, Mon. Not. R. Astr. Soc. 407, 355 (2010).

[45] W. Braun and K. Hepp, Commun. Math. Phys. 56, 125 (1977).

[46] R. Balescu, Statistical Dynamics - Matter out of Equilibrium, Imperial College Press (London, 1997).

[47] A. Lenard, Ann. Phys. 3, 490 (1960);

[48] R. L. Liboff, Kinetic Theory - Classical, Quantum, and Relativistic Descriptions, 3rd ed, Springer-Verlag (New York, 2003).

[49] P.-H. Chavanis, J. Stat. Mech. P05019 (2010).

[50] P.-H. Chavanis, Eur. Phys. J. Plus 127, 19 (2012).

[51] P.-H. Chavanis, cond-mat.stat-mech: 1303.0998.

[52] P.-H. Chavanis, cond-mat.stat-mech: 1303.1004.

[53] S. Chandrasekhar, Astrophys. J. 99, 47 (1944).

[54] O. C. Eldridge and M. Feix, Phys. Fluids 6, 398 (1963).

[55] B. B. Kadomtsev and O. P. Pogutse, Phys. Rev. Lett. 25, 1155 (1970).

[56] M. M. Sano, J. Phys. Soc. Japan 81, 024008 (2012).

[57] T. M. Rocha Filho, M. A. Amato and A. Figueiredo, in preparation.

[58] J. Dawson, Phys. Fluids 7, 419 (1964).

[59] J. L. Rouet and M. R. Feix, Phys. Fluids B 3, 1830 (1991). 
[60] W. Ettoumi and M.- C. Firpo, Phys. Rev. E 87, 030102(R) (2013).

[61] T. M. Rocha Filho, Comp. Phys. Comm. 185, 1364 (2014).

[62] M. Kac, G. Uhlenbeck and P. Hemmer, J. Math. Phys 4, 216 (1963).

[63] L. Bernardin, P. Chin, P. DeMarco, K. O. Geddes, D. E. G. Hare, K. M. Heal, G. Labahn, J. P. May, J. McCarron, M. B. Monagan, D. Ohashi and S. M. Vorkoetter, Maple Programming Guide, Maplesoft, a division of Waterloo Maple Inc. (Toronto, 2013). 